\title{
Subluxación sacroilíaca en un equino criollo colombiano
}

\author{
Gaona $\mathrm{L}^{1}$, Cruz $\mathrm{A}^{1}$, Duque-Muñoz $\mathrm{LG}^{2 *}$
}

1. Universidad Pedagógica y Tecnológica de Colombia.

2. Corporación colombiana de investigación agropecuaria, Agrosavia, Colombia.

* Correspondencia: Luis Gabriel Duque. Instituto. Corporación colombiana de investigación agropecuaria Agrosavia, $\mathrm{Cl}$ La Libertad km 17 vía puerto López, Villavicencio-Meta, Colombia.

E-mail: Igduque@agrosavia.co

Recibido: 25 Junio 2021. Aceptado: 1 Octubre 2021. Disponible en línea: 19 Noviembre 2021

Editor: P. Beldomenico

RESUMEN. La subluxación sacroilíaca es una enfermedad claudiocógena de los miembros posteriores que se puede presentar de forma aguda o crónica. La causa de esta patología se debe principalmente a resbalones, tropezones o caídas de los miembros posteriores, el diagnóstico es difícil de realizar debido a la profunda ubicación anatómica de la articulación, métodos como la ecografía y pruebas de estrés entre otros, son los procedimientos más usados en el diagnóstico de la subluxación sacroiliaca. Por tratarse de una alteración que fácilmente puede confundirse con otras condiciones patológicas de la columna, la pelvis y/o de los miembros posteriores, el diagnóstico muchas veces se realiza por eliminación de otras causas de cojera. Los tratamientos convencionales aún se proponen en la literatura, sin embargo, cada vez como opción para el tratamiento de esta patología. El presente reporte describe el caso de un equino criollo que padeció una subluxación sacroiliaca aguda del lado derecho, la cual fue diagnosticada con un examen clínico que incluía ecografía percutánea y transrectal, y tratada con infiltraciones ecoguiadas de corticoides y aspirado de médula ósea. Un mes después de la última terapia, el caballo mostró una mejoría clínica en un $80 \%$ a $90 \%$ aproximadamente y retoma actividad física controlada.

SUMMARY. Sacroiliac subluxation in a horse in Colombia. Sacroiliac subluxation is a claudiogenic disease of the hind limbs that can present acutely or chronically. The cause of this pathology is mainly due to slipping, tripping, or falling of the hind limbs, the diagnosis is difficult to make due to the deep anatomical location of the joint, methods such as ultrasound and stress tests among others, are the procedures most used in the diagnosis of sacroiliac subluxation. As it is an alteration that can easily be confused with other pathological conditions of the spine, pelvis and / or the hind limbs, the diagnosis is often made by eliminating other causes of lameness. Conventional treatments are still proposed in the literature, however regenerative therapy is increasingly used as an option for the treatment of sacroiliac joint injuries. This report describes the case of a Creole horse that suffered an acute sacroiliac subluxation on the right side, which was diagnosed with a clinical examination that included percutaneous and transrectal ultrasound and treated with ultrasound-guided infiltrations of corticosteroids and cells of the bone marrow of the sternum without processing. One month after the last therapy, the horse showed an $80 \%$ to $90 \%$ recovery and resumed controlled physical activity.

Palabras clave: Subluxación, Articulación sacroiliaca, terapia regenerativa

Keywords: Subluxation, Sacroiliac joint, regenerative therapy

\section{Introducción}

La región pélvica es una estructura semiflexible que puede presentar una pequeña deformación de las estructuras óseas, ligamentosas y musculares durante el movimiento (Hauser, 2008). El peso de la parte caudal de la columna vertebral está suspendida por los ligamentos sacroilíacos, los cuales funcionan de manera similar a un cabestrillos (Haussler, 2003). La articulación sacroiliaca está conformada por las superficies articulares de las alas del íleon y sacro, estas superficies son irregulares y están cubiertas por un delgado cartílago hialino, la cápsula articular es estrecha y está unida a los bordes articulares (Getty, 1982); directamente involucrada con la transferencia de fuerzas de propulsión proveniente de los miembros posteriores hacia la columna vertebral (Barstow y Dyson, 2015). El volumen de líquido sinovial que se encuentra en su interior es de 0,5 a $1 \mathrm{ml}$, pudiendo variar con el peso y edad del animal (Lorenz y Brounts, 2012). La capsula articular se encuentra reforzada por un estrecho ligamento sacroiliaco ventral el cual rodea la articulación, otros ligamentos que complementan esta articulación son el ligamento sacroilíaco dorsal y sacrotuberal ancho. El primero, se divide en una porción dorsal y una lateral, la dorsal se inserta sobre la tuberosidad sacra y procesos espinosos del sacro, y la lateral se inserta en la superficie caudal de la tuberosidad sacra y cresta sacra lateral (Engeli y Yeager, 2006). Lo principales músculos que contribuyen a la estabilidad de la región sacroiliaca son, los músculos glúteos, medio y superficial, y sus respectivas fascias. En 
la cara ca udal se encuentran los músculos semimembranoso y semitendinoso, y en la parte ventral de la pelvis están los músculos psoas, todos los cuales contribuyen de manera importante a la estabilidad. En condiciones normales, los músculos de esta región mantienen la movilidad de las articulaciones sacroilíacas dentro de sus límites físicos (Wessum, 2020).

Las luxaciones o dislocaciones implican la perdida de la integridad de uno o más ligamentos, así como también lesión en otras estructuras articulares, tales como la capsula articular fibrosa y tendones adyacentes. Las subluxaciones se definen como una luxación parcial (Stashak, 2003). Entre las causas de la subluxación sacroiliaca están la flacidez de sus músculos estabilizadores, esto a su vez puede originar insuficiente protección ante traumatismos como resbalones 0 caídas que pueden lesionar componentes articulares. Tres son las lesiones más frecuentes en la subluxación sacroiliaca: 1) daños en los ligamentos sacroilíacos dorsales, 2) daños en los ligamentos sacroilíacoventrales y 3) osteoartritis, esta última solo ocurre entre el $5-10 \%$ de los casos. Entre las causas del motivo de consulta de esta patología se encuentran bajo rendimiento del caballo y dificultad para mantener el peso en uno de los miembros posteriores cuando se está herrando el animal. Los caballos con subluxación sacroiliaca pueden evidenciar una variedad de hallazgos clínicos en los miembros posteriores como: disminución de la fase craneal del paso unilateral o bilateralmente, asimetría de una de la región del anca con atrofia muscular (Baxter, 2020) disminución de la propulsión en los miembros posteriores, dificultad en la transición del trote al galope y asimetría en la posición de las tuberosidades sacras (Jeffcot, 2009).

El ultrasonido es la técnica diagnóstica más sensible y no invasiva disponible para evaluar los ligamentos sacroilíacos y sus inserciones óseas en caballos, el examen es relativamente fácil de realizar y se puede utilizar equipo ecográfico estándar. Para diferenciar entre enfermedad sacroilíaca articular y desmitis del ligamento sacroilíaco, los hallazgos ecográficos deben ser interpretados junto con exámenes físicos exhaustivos y de cojera, pruebas de estrés, analgesia sacroiliaca diagnóstica y gammagrafía nuclear (Engeli, 2004). La palpación y presión sobre estructuras óseas como apófisis espinosas y tuberosidades sacras se usa para evidenciar asimetría, dolor y/o mala alineación, la palpación de la tuberosidad coxal y tuberosidad isquiática contribuyen a diferenciar entre una lesión sacroiliaca de una cojera por fractura pélvica. El test de estrés lateral o dorsoventral de los ligamentos sacroilíacos consiste en originar una respuesta dolorosa cuando se aplica presión sobre diferentes componentes óseos pélvicos y coxígeos (Haussler y Jeffcot, 2014), sin embargo aunque estos test pueden originar dolor en la región sacro iliaca no son totalmente específicos debido que otras patologías como fracturas incompletas del ala del íleon también puede originar una respuesta dolorosa. Los caballos gravemente afectados pueden reaccionar de forma exagerada ante estas pruebas de estrés flexionando los miembros posteriores repentinamente y hasta incapacidad para soportar peso en sus miembros traseros, por lo que, si se aplica excesiva presión sobre las tuberosidades sacras, el animal puede llegar a colapsar sobre sus miembros posteriores por el excesivo dolor, debido a esto es aconsejable aplicar la presión gradualmente (Haussler, 2011).

La ecografía percutánea dorsal con sonda lineal es muy útil para evaluar los ligamentos sacroilíacos dorsales y su inserción en el tubérculo sacro, los cortes transversales a nivel de las tuberosidades sacras se usan para comparar el tamaño de la parte dorsal ligamentos sacroilíacos (Wessum, 2020). Los cortes de eje largo permiten evaluar la integridad de la conformación en las fibras longitudinales de los ligamentos. Las partes laterales de los ligamentos sacroilíacos dorsales se pueden observar cómo estructuras ecogénicas delgadas que se adhieren al borde lateral del sacro, pero rara vez se detectan estos cambios clínicamente significativos. En cuanto la ecografía transrectal, es una técnica limitada en la que los cambios que se puedan presentar sean identificables fácilmente. (Head, 2014).

La ubicación profunda y poco accesible de la articulación sacroiliaca y de estructuras neurovasculares que atraviesan la cara caudal de esta articulación, han limitado la aplicación de inyecciones intra o periarticulares (Engeli y Haussler, 2011) es por lo anterior que varios autores han recomendado el uso de la guía ecográfica para ayudar a visualizar la inserción, avance y dirección de la aguja hacia la articulación sacroiliaca y a su vez prevenir la punción o irritación de las estructuras neurovasculares adyacentes (Denoix y Jacquet, 2008).

Para el tratamiento de la subluxación sacroiliaca equina se han usado perfusiones locales con soluciones anestésicas o antiinflamatorias con fines diagnósticos y/o terapéuticos (Haussler, 2011) sin embargo los resultados de estas terapias convencionales a menudo son insatisfactorios debido a la escasa capacidad de regeneración del tejido en los equinos (Dowling et al., 2000). En los últimos años el enfoque de la ciencia médica ha pasado de la reparación a la regeneración, pero el panorama de los estudios clínicos en el área de la medicina veterinaria aún es bastante limitado (Mocchi et al., 2020). Por otro lado, la aplicación de terapias basadas en células madre para tratar la desmitis del ligamento suspensorio en equinos se usó por primera vez por Herthel (2002) donde se inyectaron grandes volúmenes $(20$ a $60 \mathrm{ml}$ ) de aspirado de médula ósea esternal sin tratar en el ligamento lesionado. Este informe pertenece a una serie de casos no controlados y no aleatorizados, donde se confirma de acuerdo con los reportes de ese congreso que la técnica pareció mejorar las tasas de retorno a la función atlética en comparación a las terapias convencionales, en donde se 
reportan da tos clínicos alentadores con terapias de aspirado de médula ósea esternal no publicados.

\section{Caso clínico}

Equino entero raza criolla, de 6 años con $360 \mathrm{~kg}$ de peso y ubicado en el municipio de combita (Boyacá, Colombia), el tipo de alojamiento es en pesebrera y es ejercitado con montador día de por medio, al final de cada sesión, el caballo es conducido a ejercicios en piscina. El equino, ingresa a la Clínica de Grandes Animales de la Universidad Pedagógica y Tecnológica de Colombia, donde se informa que el equino sufrió un resbalón al entrar de la piscina, quedando como secuela, andar envarado del tren posterior con una cojera 4 de 5 en el miembro posterior derecho, asimetría de la región del anca con descenso del lado derecho (Figura 1A); dolor moderado al aplicar presión digital en la región sacra y lumbosacra; se hizo una prueba que consistió en empujar la tuberosidad sacra ipsilateral con dirección hacia el plano medio y al mismo tiempo se haló la base de la cola lateralmente, esta prueba dió como resultado una flexión refleja del tren posterior como respuesta al dolor originado.

Se realiza pruebas diagnósticas con ecógrafo y un transductor tipo lineal sobre la región glútea, alas del ilion, región sacra y lumbosacra, además se realiza ecografía transrectal para observar la articulación lumbosacra a nivel de la unión de las alas y cuerpos vertebrales, hueso sacro ventralmente y arterias iliacas; no fue posible evaluar la articulación sacroiliaca por técnica transrectal. Al terminar el examen ecográfico, se evidenció leve pérdida de la ecogenicidad de la porción dorsal del ligamento sacroilíaco. Se llega a la conclusión que, se presentó una leve disminución por el conjunto de hallazgos clínicos y el descarte de lesiones en otros sitios anatómicos, el paciente tiene una subluxación sacroilíaca derecha con leve ecogenicidad de la porción dorsal de ligamento sacroilíaco derecho, se realizó un diagnóstico clínico apoyado por las imágenes correspondientes a la lesión.

El tratamiento se dividió en 3 sesiones y al comienzo de cada una de estas se manejó un protocolo de neuroleptoanalgesia basada en $180 \mathrm{mg}$ de xilacina (10\%), 11 mg de acepromacina (1\%) y $540 \mathrm{mg}$ de tramadol (5\%), esto con el fin de inducir analgesia, tranquilización y disminuir el movimiento del animal durante los procedimientos. En la primera sesión administraron 12 $\mathrm{mg}$ de dexametasona $(2,63 \mathrm{mg} / \mathrm{mL})$ con agujas calibre 21 por $1^{1 / 2}$ pulgadas en tres puntos sobre la porción dorsal del ligamento sacroilíaco, se continua con un abordaje periarticular craneal ecoguiado usando una aguja espinal calibre 18 de $20 \mathrm{cms}$ de longitud y se administra $18 \mathrm{mg}$ de triamcinolona $(10 \mathrm{mg} / \mathrm{mL})$. Dos semanas después se realizó una segunda sesión que consistió en aspirar contenido de la medula ósea de la 5 esternebra con ayuda de ecografia, una vez obtenidos aproximadamente $30 \mathrm{ml}$ del contenido medular disueltos en una solución de heparina, se prosigue con la filtración del contenido en un equipo de transfusión para finalmente ser infiltrado de forma periarticular craneal, similar al primer procedimiento (Figura 2). En la última sesión (tercera sesión) se repite el procedimiento anterior.

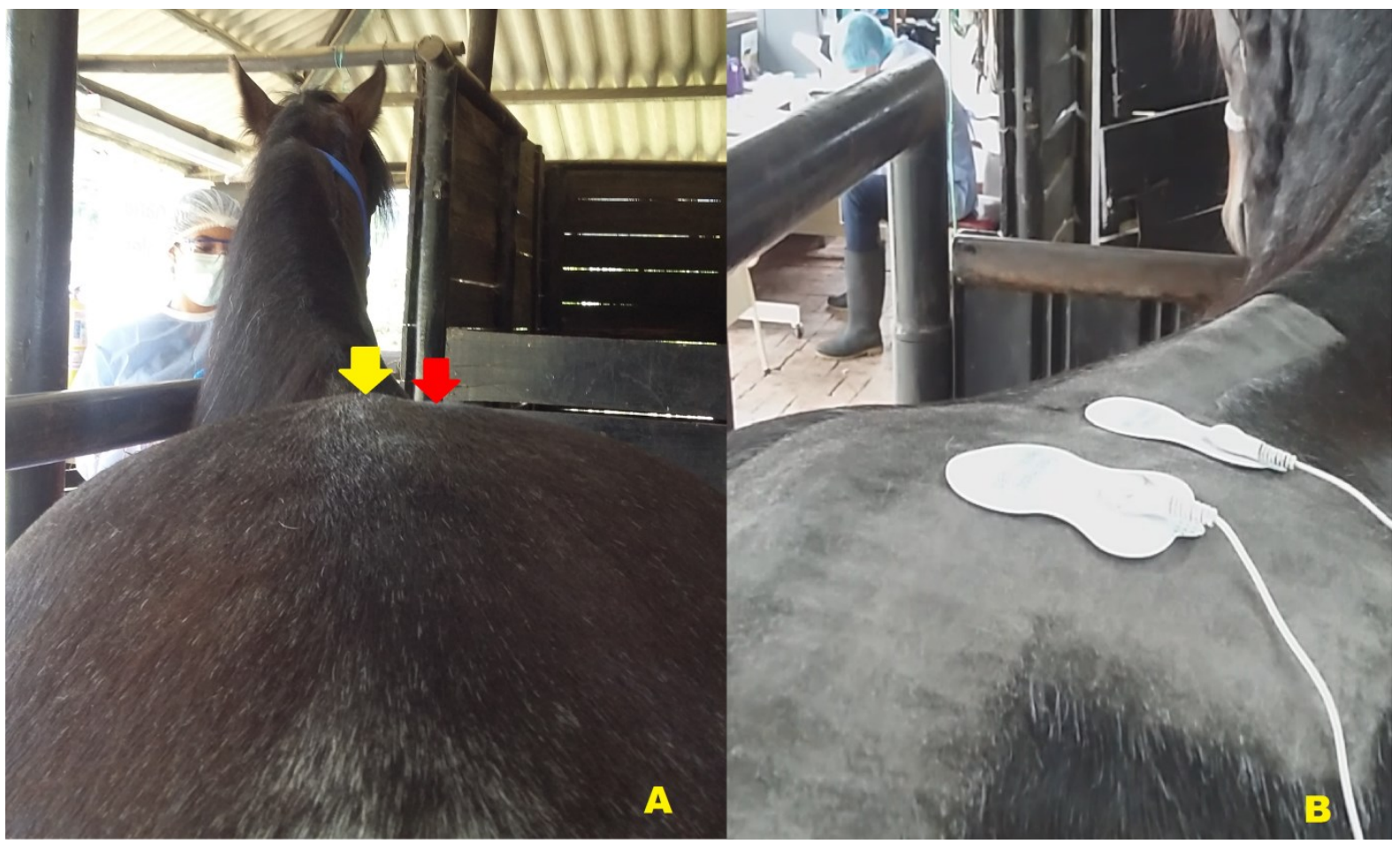

Figura 1. Asimetría de la región del anca (A), ubicación de los electrodos (B). Nivel de la tuberosidad sacra izquierda (flecha amarilla), nivel de la tuberosidad sacra derecha (flecha roja) 
El manejo de la fisioterapia consistió en 30 minutos diarios de actividad física esta rutina se llevó a cabo en dos sesiones así: una en la mañana y una en la tarde en la cual realizaban caminatas sobre un terreno inclinado o con postes sobre el suelo para que estos sirvieran obstáculo. Adicionalmente a esta rutina, se realizaron sesiones diarias (mañana y tarde) que consistían en aplicar electroestimulación local con dos electrodos en la región sacra y glútea (Figura 1 B), el equipo se calibro con un tiempo de 15 minutos y una intensidad de 3 donde 0 era sin estímulos eléctricos y 15 era la máxima potencia eléctrica a través de la aplicación de corriente galvánica exponencial de baja intensidad (máx. 3mA) suministrada. La intensidad y tiempo de todas las actividades realizadas durante la fueron incrementadas moderadamente de forma gradual.

Un mes después a la última sesión, el caballo mostró recuperación de un $80 \%$ a $90 \%$, el equino fue retirado de las instalaciones de la clínica y actualmente se encuentra realizando actividad física controlada con montador.

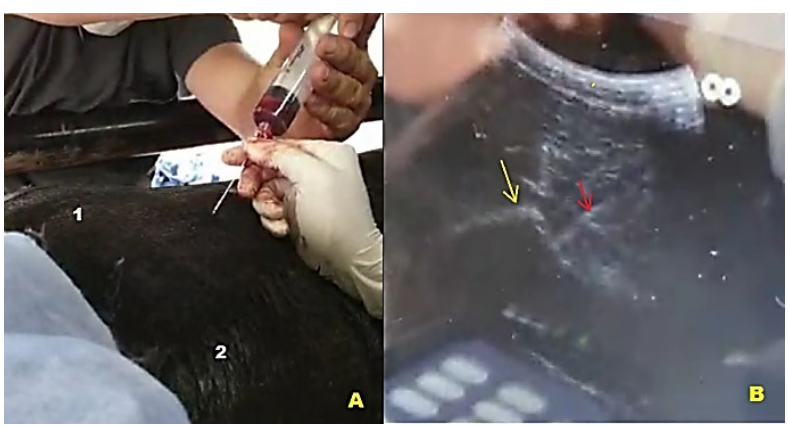

Figura 2. Sitio de punción (A), fotografía de la infiltración ecoguiada (B). 1) región de la tuberosidad sacra; 2) región de la tuberosidad coxal, borde dorsocraneal del ala del ílion (flecha amarilla), hiperecogenicidad perteneciente a la aguja espinal (flecha roja).

\section{Discusión}

Desde el momento que el animal fue desembarcado se sospechó de subluxación sacroilíaca por manifestar cambios musculoesqueléticos característicos de la enfermedad; sin embargo, debido que no fue posible evidenciar lesiones en articulación sacroiliaca por ecografía transrectal, fue necesario basarse en un proceso de eliminación de otras causas que originasen dolor en el miembro afectado (Hendrickson, 2003), lo que justificó realizar exámenes con ultrasonido en regiones distintas a la sacroiliaca.

En este paciente se decidió no realizar una terapia convencional con AINEs en razón a que la misma debe ser prolongada (14 a 21 días) (Hendrickson, 2003) y esto genera alto riesgo de desencadenar lesiones gástricas, del colon o bien insuficiencia renal, por lo que se prefiere hacer inyecciones locales con corticosteroides alrededor de la región de la articulación sacroilíaca, estos procedimientos se han utilizado de manera extensa en la clínica equina (Haussler y Jeffcot, 2014). La dosis periarticular de triamcinolona administrada en este caso es soportada por un informe de Genovese (1983), donde se demostró que la administración de triamcinolona a una dosis máxima de $18 \mathrm{mg}$ no origina laminitis en 1200 caballos tratados. Otro estudio demostró que incluso usada la triamcinolona a dosis que oscilaban entre los 20 y $45 \mathrm{mg}$ de dosis total, originaba laminitis solo en el $0,15 \%$ de los casos (Bathe 2007). Esto demuestra que aparte de tener un efecto condroprotector (Mcllwraith, 2010), la dosis de triamcinolona administrada en este caso disminuye el riesgo de originar laminitis.

La obtención de contenido medular extraído directamente de las dos últimas esternebras sin procesar en laboratorio y aplicarlo directamente en la lesión, es un procedimiento que Herthel (2002) realizo a un grupo de caballos, y al igual que el presente caso se obtuvo buen grado de recuperación con retorno a la función atlética. Con respecto a los resultados de recuperación obtenidos, aún queda la interrogante si la recuperación de la subluxación sacroiliaca se debió a las células madre mesenquimales que se encuentran en el aspirado esternal o a otros componentes que se obtienen por esta técnica. Según Fortier (2011), es poco probable que los resultados observados por Hertel se debieran a las células madre, pues se sabe que se obtienen pocas células madre en los aspirados de médula ósea y además indica que cualquier efecto clínico puede atribuirse a las numerosas sustancias bioactivas como los factores de crecimiento que producen las plaquetas. Si bien las células madre que se obtienen del esternón no son suficientes para obtener un efecto regenerativo adecuado, si es claro que su obtención directa y su inmediata aplicación sin procesar es una ventaja para los casos que no se tenga al alcance u laboratorio especializado, además es una técnica económica que solo requiere un ecógrafo como herramienta especializada.

Con respecto a la fisioterapia establecida en este caso, Clayton (2014) afirma que el trabajo con postes a nivel del suelo o elevados se utilizan en programas de rehabilitación en caballos. Además, este tipo trabajo mejora o restaura los rangos de movimiento de las articulaciones y fortalecen los músculos propulsores (Clayton, 1991). Por otro lado, Dainty (2016) expone que el trabajo en terreno inclinado puede ser beneficioso para rehabilitar lesiones pélvicas y mejorar el reclutamiento de grupos musculares y a su vez fortalecerlos. Finalmente, lo que concierne a la electroterapia, primero que todo se debe proporcionar suficiente energía para superar el umbral de respuesta fisiológica en el tejido (Watson y Lawrence 2016), de forma similar en este caso se pudo evidenciar una respuesta contráctil del musculo gran dorsal y glúteo medio. Según Watson y Lawrence (2016) esta respuesta fisiológica puede proporcionar un efecto terapéutico en la reparación de tejidos, función muscular y tratamiento del dolor. Aunque los efectos terapéuticos con 
electroterapia prometen una amplia aplicabilidad en la medina veterinaria, es claro que en la práctica se trata de un campo relativamente nuevo en equinos y que por ello deben diseñarse correctamente métodos de estimulación eléctrica que los animales toleren al igual que los humanos (Sheila y Schils, 2009). En concordancia con lo anterior, al comparar este caso con otros caballos tratados con electroterapia, se pudo observar un grado de tolerancia menor. Esto indica que la estandarización de estos protocolos en equinos aun es difícil y que el grado de aplicabilidad en esta especie puede depender de cada individuo, por lo que se requiere aplicar esta terapia a un mayor número de caballos para tener resultados más robustos.

A pesar de que quedan dudas sobre si son otros componentes medulares y no las células madre mesenquimales las responsables de favorecer la recuperación en este caso, la técnica de extraer células de la medula ósea y aplicarlas sin procesar de forma periarticular craneal en la articulación sacroiliaca, es un procedimiento que se puede realizar en campo con materiales limitados y buena recuperación de los pacientes.

La recuperación de la subluxación sacroiliaca aguda es posible, combinando diferentes técnicas terapéuticas de la manera adecuada: reposo, infiltraciones regionales con corticoides, terapia regenerativa $y$ fisioterapias.

El uso de la ecografía en el diagnóstico y tratamiento de la subluxación sacroiliaca es indispensable, sin embargo, esta técnica diagnóstica requiere horas de práctica y conocimientos anatómicos detallados para ser efectiva.

\section{Referencias}

Barstow A, Dyson S. 2015. Clinical features and diagnosis of sacroiliac joint region pain in 296 horses 2004-2014. Eq. Vet. Educ. 12: 637-647.

Bathe AP. 2007. The corticosteroid laminitis story: The clinician's viewpoint. Eq. Vet. J. 39: 12-13.

Clayton HM, Stubbs NC, Lavagnio M. 2014. Stance phase kinematics and kinetics of horses trotting over poles. Eq. Vet. J. 47: 113- 118.

Clayton HM. 1991. Suppling exercises. En: Conditioning Sport Horses. Sport Horse Publications, Canada. Pp. 121-134.

Dainty E. 2016. Assessment and treatment techniques of the equine thoracolumbar spine, pelvis and pelvic limb En: McGowan CM, Goff L, eds. Animal physiotherapy assessment, treatment and rehabilitation of animals. $2^{\mathrm{a}}$ ed. WileyBlackwell, Chichester. Pp 313- 327.

Denoix JM, Jacquet S. 2008. Ultrasound-guided injections of the sacroiliac area in horses. Eq. Vet. Educ. 20: 203-207.
Dowling BA, Dart AJ, Hodgson DR, Smith RK. 2000. Superficial digital flexor tendonitis in the horse. Eq. Vet. J. 32: 369-378.

Engeli E, Haussler K. 2011. Review of injection techniques targeting the sacroiliac region in horses. Eq. Vet. Educ. 24: 529541.

Engeli, E, Yeager A. 2006. Ultrasonographic technique and normal anatomic features of the sacroiliac region in horses. Vet. Radiol. Ultrasound 47: 391-403.

Engeli E, Yeager A, Haussler K. 2004. Use and limitations of ultrasonography in sacroiliac disease. En: AAEP Annual Convention. Denver: American Association of Equine Practitioners.

Fortier LA, Travis AJ. 2011. Stem cells in veterinary medicine. Stem Cell Res. Therap. 2: 9.

Genovese RL. 1983. The use of corticosteroids in racetrack practice. En: Proceedings symposium effective use of corticosteroids in veterinary practice.

Getty R. 1982. Anatomia de los Animales Domesticos. $5^{\mathrm{a}}$ ed. Barcelona: MASSON S.A.

Haussler K, Jeffcot, B. 2014. Back and pelvis. En: Hinchcliff, W., Kaneps, \& Geor, J, eds: Equine Sports Medicine and Surgery: Basic and clinical sciences of the equine athlete. $2^{2}$ ed. WileyBlackwell. Pp. 419-453.

Haussler, K., McGilvray, K. 2008. Sacroiliac joint motion and pelvic deformation in horses. Am. Assoc. Eq. Pract. 54: 258-259

Haussler K. 2011. Diagnosis and management of sacroiliac joint injuries. In: Ross W, ed. Diagnosis and Management of Lameness in the Horse. 2a ed. Elsevier, Missouri. Pp 583-591.

Head M. 2014. Ultrasonography of the pelvis. In: Kidd J, Lu K y Frazer $M$, eds. Atlas of equine ultrasonography. WileyBlackwell. Pp 183-197.

Hendrickson DA. 2003. La pelvis. En: Stashak, TS ed. Adams: Claudicacion en el caballo. $5^{\text {a }}$ ed. Buenos Aires: Inter-Medica. Pp 1123- 133.

Herthe DJ. 2002. Suspensory desmitis therapies. En: 12th symposium. American College of Veterinary Surgeons.

Jeffcot B. 2009. The Normal Anatomy of the Osseous Structures of the Back and Pelvis. In Frances $M$, eds. Equine Back Pathology: Diagnosis and Treatment. 1a ed. WileyBlackwell. Pp. 3-15.

Lorenz J, Brounts, H. 2012. Sacroiliac Injuries in Horses. Compend. Contin. Educ. Vet. 11: E3.

Mcllwraith cw. 2010. The use of intra-articular corticosteroids in the horse: What is known on a scientific basis?. Eq. Vet. J. 42: 563-571.

Mocchi M, Dotti S, Del Bue M, Villa R y Bari E. 2020. Veterinary regenerative medicine for musculoskeletal Disorders: Can mesenchymal stem/stromal cells and their secretome be the new frontier?. Cells 9: 1453.

Sheila J, Schils MS. 2009. Review of electrotherapy devices for use in veterinary medicine. Am. Assoc. Eq. Pract. 55: 68-73. 
Stashak TS. 2003. Adams: Claudicación en el caballo. InterMedica, Buenos Aires.

Watson T, Lawrence K. 2016. Electrophysical agents in animal physiotherapy En: McGowan CM, Goff L, eds. Animal physiotherapy assessment, treatment and rehabilitation of animals. 2a ed. Wiley-Blackwell, Chichester. Pp 212-223.

Wessum RV. 2020. Lameness associated with the axial skeleton. En: Baxter M, ed. Adams and Stachak's lameness in horses. $7^{\text {a }}$ ed. Wiley-Blackwell, New jersey. Pp. 763-798. 